\title{
Stunting May Determine the Severity of Malaria-Associated Anemia in African Children
}

\author{
Hans Verhoef, $\mathrm{PhD}^{*} \ddagger$; Clive E. West, $\mathrm{PhD}, \mathrm{DSc}^{*} \S$; Jacobien Veenemans, $\mathrm{MD}^{*}$; Yves Beguin, $\mathrm{PhD} \|$; and \\ Frans J. Kok, PhD*
}

\begin{abstract}
Objective. Evidence from previous studies that malnourished children are protected against malaria is controversial. In individuals repeatedly exposed to malaria, immunity may develop first against severe disease, then against pyrogens, and last, against parasites. If this is true, this would suggest that reduced immune function that may exist in stunted children exacerbates the severity of malarial signs and symptoms, rather than the occurrence of parasitemia. On the other hand, several studies have suggested that malnourished children are protected to some degree against malaria. Our aim was to evaluate whether observational data support the hypothesis that nutritional inadequacies that cause stunting modify the associations between malaria and hematologic indicators such as hemoglobin concentration and serum concentrations of C-reactive protein and soluble transferrin receptor (sTfR). We showed earlier that increased serum concentrations of these receptors in asymptomatic malaria may be explained, at least in part, by increased erythropoiesis to compensate for malaria-induced hemolysis.
\end{abstract}

Methodology. Community-based cluster survey among Kenyan children aged 2 to 36 months asymptomatic for malaria or anemia $(n=318)$.

Results. When adjusted for age and wasting, the malaria-associated decrease in mean hemoglobin concentration was $8.5 \mathrm{~g} / \mathrm{L}$ and $15.8 \mathrm{~g} / \mathrm{L}$ in nonstunted and stunted children, respectively. The malaria-associated increase in geometric mean serum concentrations of sTfR was 1.1fold and 1.8-fold in nonstunted and stunted children, respectively. The malaria-associated increase in geometric mean serum concentrations of $\mathrm{C}$-reactive protein was 1.4-fold and 2.3-fold in nonstunted and stunted children, respectively. Thus, children with malaria and those who were stunted suffered from more severe anemia and had higher serum concentrations of C-reactive protein and STfR than would be expected from the combined effect of the 2 working independently.

Conclusions. Our results are consistent with the notion that the nutritional inadequacies causing stunting also impair host immunity, thus increasing the degree to which malaria is associated with decreased concentrations of hemoglobin, with increased inflammation, and with increased iron demand in developing erythroblasts. Increased intake of micronutrients may not only reduce stunting and nutritional anemia, but also reduce malaria-

From the *Division of Human Nutrition and Epidemiology, Wageningen University, the Netherlands, ‡African Medical and Research Foundation, Nairobi, Kenya, §Department of Gastroenterology, University Medical Centre Nijmegen, the Netherlands, and \|Department of Medicine, Division of Hematology, University of Liège, Belgium.

Received for publication Jan 19, 2001; accepted Jun 13, 2002.

Address correspondence to Clive E. West, PhD, DSc, Division of Human Nutrition and Epidemiology, Wageningen University, Box 8129, 6700 EV

Wageningen, the Netherlands. E-mail: clive.west@staff.nutepi.wau.nl associated anemia. Pediatrics 2002;110(4). URL: http:// www.pediatrics.org/cgi/content/full/110/4/e48; anemia, hemoglobin, Kenya, malaria, stunting.

ABBREVIATIONS. sTfR, soluble transferrin receptor; CI, confidence interval.

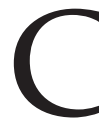

hildren in developing countries generally fail to achieve their genetically determined potential height because of poor diet and infection. ${ }^{1,2}$ It is likely that the nutritional inadequacies that cause stunting also impair host immunity, thereby increasing the incidence, severity, and duration of many infectious diseases. ${ }^{3}$

Individuals with repeated exposure to malaria are protected against severe disease and death before they develop the ability to regulate fever, parasite density, and ultimately infection in itself. This has led to the hypothesis that immunity develops first against severe disease, then against pyrogens, and lastly against parasites. ${ }^{4}$ If true, this would suggest that reduced immune function, such as that which may exist in stunted children, exacerbates the severity of malarial signs and symptoms, rather than the occurrence of parasitemia. On the other hand, several studies have suggested that malnourished children are protected to some degree against malaria. ${ }^{5-7}$

The concentration of soluble transferrin receptor (sTfR) in serum is a new indicator of iron demand by the erythroid precursor mass. It provides a measure of both the rate of erythropoiesis and the degree of iron deficiency, ${ }^{8}$ and is influenced little or not at all by the inflammatory response to infection. We recently reported increased serum concentrations of sTfR and C-reactive protein in children with asymptomatic malaria. ${ }^{9}$ These increased serum sTfR concentrations may be explained, at least in part, by increased erythropoiesis to compensate for malariainduced hemolysis. ${ }^{9}$

We conducted a cross-sectional study in children aged 2 to 36 months without symptoms of malaria or anemia living in an area with seasonal malaria transmission. The aim of our study was to evaluate whether data from this survey give support to the hypothesis that the relationship between malarial parasitemia and stunting is synergistic, ie, that the presence of asymptomatic malaria and nutritional inadequacies (as indicated by stunting) results in a lower concentration of hemoglobin, and higher serum concentrations of sTfR and C-reactive protein, 
than would be expected from the combined effect of the 2 working independently.

\section{SUBJECTS AND METHODS}

\section{Study Area}

The study was conducted in the first annual rainy season (April through June) of 1997 in an area of seasonal malaria in Eastern Province, Kenya. The study area comprises $\sim 720 \mathrm{~km}^{2}$ at an altitude of 800 to $900 \mathrm{~m}$ above sea level, located halfway between Nairobi and Mombasa on the road and rail link. Malaria infection reported at the clinical facilities in the area are attributable exclusively to Plasmodium falciparum. No malaria control program is active in the area, and no epidemiologic or entomologic studies on malaria have been conducted previously.

\section{Study Population and Sampling Procedures}

The target population comprised children living in the study area aged 2 to 36 months, with no manifestations reported by mothers or caregivers that were compatible with malaria or anemia. Few of the children studied suffer from intestinal worm infections or schistosomiasis. ${ }^{9}$ Sampling procedures have been described in detail previously. ${ }^{9}$ In brief, children were selected using a 2-stage cluster sampling procedure. At the first sampling stage, 45 of 79 communities in the study area were sampled systematically, with a sampling probability proportional to size as measured by the number of households. At the second sampling stage, 12 households were randomly selected within each selected community, and all resident children with no symptoms of malaria or anemia and within the desired age range were selected for the study.

\section{Field Procedures}

Informed consent was sought and obtained from community leaders, locally active auxiliary health workers, and parents of eligible children. Children were treated as deemed necessary on completion of the survey. The study was approved by the African Medical and Research Foundation and the Kenya Medical Research Foundation, whose ethical standards were followed.

Field staff were trained in anthropometric techniques, and measurements were standardized ${ }^{10,11}$ before data collection. Recumbent length $(<2 \mathrm{y})$ or standing height $(\geq 2 \mathrm{y})$, body weight (Seca 720 infant scale [Hamburg, Germany], allowing for measurements to be read within $10 \mathrm{~g}$ ), mid-upper arm circumference, and triceps skinfold thickness (Harpenden calipers, CMS Weighing Equipment, London, United Kingdom) were recorded as the average of duplicate measurements, respectively.

The procedures for collection, handling, and analysis of capillary blood samples has been described elsewhere. ${ }^{9}$ Hemoglobin concentrations were measured using a field meter (HemoCue, Ängelholm, Sweden) and malaria was detected by microscopic examination of Giemsa-stained blood slides. Concentrations of sTfR were measured by enzyme-linked immunosorbent assay (R\&D Systems, Minneapolis, MN). C-reactive protein concentrations were interpreted as indicators of the activity of infection and measured by a standard turbidimetric method. Intra- and interassay coefficients of variation for these biochemical tests are reported elsewhere. ${ }^{9}$

\section{Response and Missing Values}

The study included 318 children. Of 35 children who did not participate or fully participate, 14 were replaced by random selection, and weighting was used to maintain the validity of assuming an equal probability sample. ${ }^{12,13}$ Sample sizes reported below 318 are attributable to missing values.

\section{Statistical Analysis}

Height-for age and weight-for-height z-scores were calculated using Epi Info v6.04 (Centers for Disease Control and Prevention, Atlanta, GA). ${ }^{14}$ Being stunted or wasted was defined by heightfor-age or weight-for-height $z$ scores $<-2$ standard deviations of the median of the National Center for Health Statistics reference population, respectively. ${ }^{15}$ Data were explored using SPSS (v7.5 for Windows; SPSS Inc, Chicago, IL) and analyzed using SUDAAN (stand-alone software v7.5.2a for Windows; Research Triangle Institute, Research Triangle Park NC), assuming 2-stage cluster sampling with replacement at the first sampling stage. The variance estimates under this assumption do not take into account that clusters were sampled from a finite population. Thus, the standard error values and confidence intervals reported here are overestimates, and statistical tests are conservative in detecting existing associations.

As a first analytic step, the relationships between malaria and height-for-age $z$ score and between malaria and weight-for-height $z$ score were assessed by logistic regression. Serum concentrations of sTfR and C-reactive protein were normalized by decimal logarithm transformations, and their relationship with stunting or wasting were assessed by linear regression analysis. Associations between height-for-age $z$ score and age class (2-11 months, 12-23 months, >24 months) or continuous variables (weight-for-height $z$ score, hemoglobin concentration) were assessed by analysis of variance and linear regression analysis, respectively.

Multiple linear regression was used to compare stunted and nonstunted children regarding their associations between malaria and hemoglobin concentration, or between malaria and log transformed serum concentrations of sTfR or C-reactive protein, respectively. Possible interaction was tested directly by multivariate linear regression analysis. Adjustment for age class and sex led to similar conclusions and effect estimates, so that these variables were excluded from the final model.

\section{RESULTS}

The prevalence of children who were stunted or wasted was $38.7 \%(95 \%$ confidence interval $[\mathrm{CI}]$ : $32.0-45.4)$ and $4.7 \%$ (95\% CI: $1.6-7.8)$, respectively. The prevalence of malaria was $17.6 \%$ (95\% CI: 11.3 $23.8 ; n=318$ ): for age classes 2 to 11 months, 12 to 23 months, and $>24$ months, these figures were $12.6 \%$ $(n=92), 18.8 \%(n=116)$, and $20.4 \%(n=111)$, respectively, and for girls and boys they were $16.1 \%$ $(n=145)$ and $18.8 \%(n=173)$, respectively. Other characteristics of the study population, broken down by age class and sex, are given in Table 1 . Stunting was most pronounced in children aged 12 to 23

TABLE 1. Characteristics Indicating Nutritional Status of the Population Studied, by Age Class and Sex

\begin{tabular}{|c|c|c|c|c|c|c|c|c|}
\hline \multirow{2}{*}{ Age class } & \multicolumn{2}{|c|}{$\begin{array}{l}\text { Height-for-Age } \\
z \text { Score }\end{array}$} & \multicolumn{2}{|c|}{$\begin{array}{c}\text { Weight-for-Height } \\
\text { z Score }\end{array}$} & \multicolumn{2}{|c|}{$\begin{array}{l}\text { Mid-Upper Arm } \\
\text { Circumference, } \mathrm{cm}^{*}\end{array}$} & \multicolumn{2}{|c|}{$\begin{array}{l}\text { Triceps Skinfold } \\
\text { Thickness, mm* }\end{array}$} \\
\hline & & & & & & & & \\
\hline $2-11 \mathrm{mo}$ & $-1.29 \pm 1.56$ & (92) & $-0.03 \pm 0.96$ & (92) & 14.5 [13.2-15.1] & $(92)$ & $7.51[5.76-7.89]$ & (91) \\
\hline $12-23 \mathrm{mo}$ & $-2.04 \pm 0.91$ & (113) & $-0.85 \pm 0.74$ & (113) & $14.7[13.7-15.4]$ & (116) & $6.89[6.00-7.85]$ & (114) \\
\hline$>24 \mathrm{mo}$ & $-1.93 \pm 0.90$ & (107) & $-0.88 \pm 0.57$ & (107) & $14.7[14.1-15.4]$ & (108) & $6.97[6.25-7.98]$ & (107) \\
\hline \multicolumn{9}{|l|}{ Sex } \\
\hline Male & $-1.90 \pm 1.23$ & (169) & $-0.59 \pm 0.55$ & (169) & 14.8 [14.0-15.4] & $(146)$ & 7.05 [5.95-7.94] & (143) \\
\hline Female & $-1.65 \pm 0.66$ & (142) & $-0.65 \pm 0.54$ & (142) & $14.5[13.5-15.2]$ & (135) & $6.93[5.96-7.92]$ & (134) \\
\hline All & $-1.79 \pm 0.81$ & (311) & $-0.62 \pm 0.42$ & (311) & $14.6[13.8-15.3]$ & (316) & $6.97[5.95-7.95]$ & (311) \\
\hline
\end{tabular}

Mean \pm standard deviation or *median [interquartile range] $(n)$.

Figures were derived from weighted analysis and were rounded off to integer values. 
months, whose height-for-age $z$ score was 0.75 below the value of children aged 2 to 11 months.

An increase of one unit $z$ score of height-for-age was associated with an odds ratio of malaria of 0.87 (95\% CI: $0.69-1.09 ; P=.23$ ), a weight-for-height increase of $0.16 z$ scores (95\% CI: $0.04-0.27 ; P=.009$ ), and a hemoglobin concentration increase of $2.0 \mathrm{~g} / \mathrm{L}$ (95\% CI: $0.7-3.4 \mathrm{~g} / \mathrm{L} ; P=.004)$. Each increase of 1 unit $z$ score of weight-for-height was associated with an odds ratio of malaria of 0.78 (95\% CI: $0.58-1.05$; $P=.10)$ and an increase in hemoglobin concentration of $2.3 \mathrm{~g} / \mathrm{L}$ (95\% CI: $1.0-3.6 \mathrm{~g} / \mathrm{L} ; P=.001)$, respectively. When adjusted for wasting (weight-for-height $z$ score quartiles), an increase of 1 unit $z$ score of height-for-age corresponded to an odds ratio of malaria of 0.93 (95\% CI: $0.73-1.17 ; P=.52)$. When analyzed by univariate linear regression techniques, there was no evidence of a biologically or statistically significant relationship between stunting and $\log$ transformed serum concentrations of sTfR or C-reactive protein, or between wasting and log transformed serum concentrations of sTfR or C-reactive protein (data not shown).

Univariate analysis showed that children with malaria had lower mean hemoglobin concentrations than children without malaria $(92.7 \mathrm{~g} / \mathrm{L}$ compared with $104.1 \mathrm{~g} / \mathrm{L}$; difference $11.3 \mathrm{~g} / \mathrm{L}$; 95\% CI: $6.4-16.3$ $\mathrm{g} / \mathrm{L} ; P=.0001)$. Children with malaria also had higher geometric mean serum concentrations of sTfR (11.4 mg/L compared with $7.8 \mathrm{mg} / \mathrm{L}$; ratio $1.4 ; 95 \%$ CI: $1.1-1.9 ; P=.005)$ and C-reactive protein $(12.5$ $\mathrm{mg} / \mathrm{L}$ compared with $6.8 \mathrm{mg} / \mathrm{L}$; ratio $1.8 ; 95 \% \mathrm{CI}$ : $1.2-2.7 ; P=.004)$.

Figure 1 compares stunted and nonstunted children regarding their association between malaria and hematologic indicators. There was no evidence for substantial effects of stunting on these indicators in the absence of malaria. The malaria-associated decrease in mean hemoglobin concentration was 8.6 g/L (95\% CI: 2.6-14.6 g/L) in nonstunted children, and $16.4 \mathrm{~g} / \mathrm{L}$ (95\% CI: 9.3-23.5 g/L) in stunted children (Fig 1, top). In nonstunted children, geometric mean serum concentrations of sTfR were 1.2-fold (95\% CI: 0.9-1.6) higher in children with malaria than those without malaria (Fig 1, center); in stunted children, this ratio was 1.8 (95\% CI: 1.3-2.5). Among nonstunted children, geometric mean serum concentrations of C-reactive protein were 1.4 -fold $(95 \% \mathrm{CI}$ : 0.8-2.3) higher in children with malaria than those without malaria (Fig 1, bottom); among stunted children, this ratio was 2.3 (95\% CI: 1.2-4.7).

These relationships were further assessed by multivariate regression analysis, which was used to test the significance of product terms directly and to adjust for possible confounding by age class and wasting (entered into the model as weight-for-height $z$ score quartiles). When so analyzed, the malariaassociated decrease in mean hemoglobin concentration was $8.5 \mathrm{~g} / \mathrm{L}$ and $15.8 \mathrm{~g} / \mathrm{L}$ in nonstunted and stunted children, respectively ( $P$ value of test for difference: .08). The malaria-associated increase in geometric mean serum sTfR concentrations was 1.1fold and 1.8-fold in nonstunted and stunted children, respectively $(P$ value of test for difference $=.05)$. The
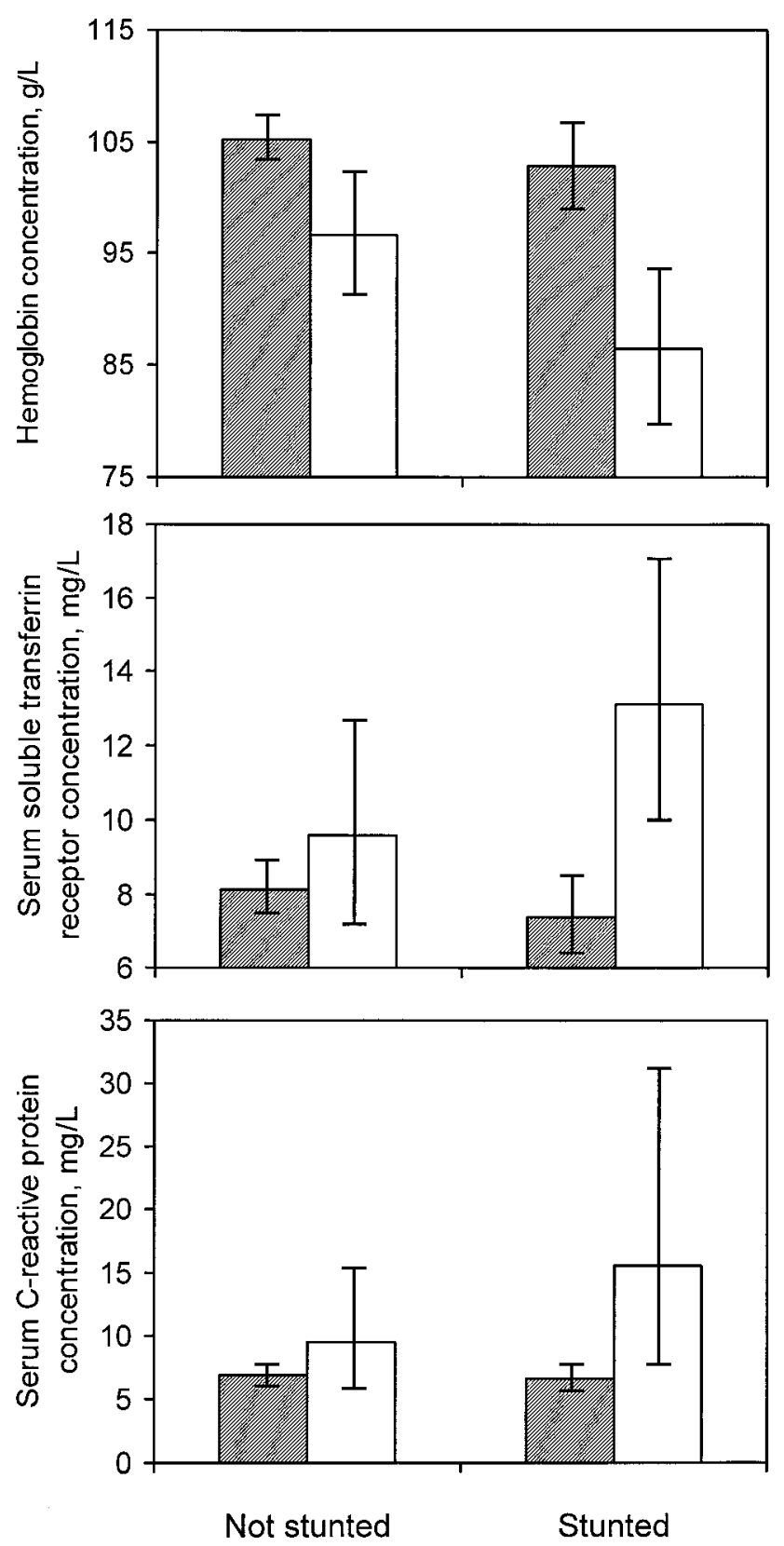

Fig 1. Modification by stunting of the relation between malaria and mean hemoglobin concentration (top; $n=311$ ), and geometric mean serum concentrations of transferrin receptor (center; $n=$ 222 ) and C-reactive protein (bottom; $n=90$ ). When adjusted for age and wasting, $P$ values indicating significance of the malariaassociated differences in these hematologic indicators were .08 .05 , and .05 , respectively (see text). Absence and presence of malaria are indicated by black and white columns respectively; error bars indicate $95 \%$ CIs.

malaria-associated increase in geometric mean serum concentrations of C-reactive protein was 1.4fold and 2.3-fold in nonstunted and stunted children, respectively ( $P$ value of test for difference: .05$)$. These effect estimates are similar for nonadjusted estimates (see previous paragraph), indicating little or no confounding by age or wasting. There was no evidence that the relationship between malaria and these hematologic indicators was modified by age (data not shown).

Missing data were mostly attributable to insuffi- 
cient serum being available to determine concentrations of sTfR and serum C-reactive protein. Children with missing data $(n=96)$, and who were therefore excluded from the multiple regression analysis, did not have substantially different hemoglobin concentrations or height-for-age $z$ scores from those who were included (not shown), although fewer of them suffered from malaria (14\% compared with 25\%). Selection bias would have occurred if the observed relationships were different in children with missing values.

\section{DISCUSSION}

Our findings support the hypothesis that in stunted children, malaria is associated with lower hemoglobin concentrations and higher serum concentrations of sTfR and C-reactive protein than in their nonstunted counterparts. There is no clear evidence that stunting is associated with an increased prevalence of malarial infection.

The prevalences of stunted and wasted children were $39 \%$ and $5 \%$, respectively. The mean hemoglobin concentration was $102 \mathrm{~g} / \mathrm{L}$, and we showed earlier that the prevalence of anemia in this population was $71 \% .{ }^{9}$ These values are typical for this age group in Kenya and other countries in Africa. ${ }^{16-19}$ Our study confirmed that stunting was most pronounced in children aged 12 to 23 months. This corroborates findings in most studies that stunting in developing countries peaks at the age of 24 months and stabilizes thereafter. ${ }^{20}$ Missing values occurred mainly because of insufficient volume of serum being available for biochemical analysis: there is no reason to assume that this led to selection bias in the relationships observed. Our conclusions may therefore apply to African preschool children who are asymptomatic for anemia and malaria and living in areas of seasonal malaria.

Serum sTfR concentrations are closely correlated to the number of receptors expressed on the surface of erythroblasts, where they transport transferrinbound iron into the cell. An expansion of the erythroid mass results in increased serum sTfR concentrations, and increased transferrin receptor expression takes place on the surface of iron-deficient erythroblasts. Serum sTfR concentrations thus reflect both the rate of erythropoiesis and the degree of iron deficiency in the erythron. ${ }^{8}$ Serum sTfR concentrations are not or only marginally influenced by the inflammatory response to infection, ${ }^{8,21}$ and no infections investigated so far except for malaria have been shown to be associated with serum sTfR concentrations. We proposed earlier that the observed increase in serum sTfR concentration in malaria is attributable entirely or in part to hemolysis, which occurs in malaria but is not a common feature in other infections. ${ }^{9}$ Malaria-induced hemolysis leads to lower hemoglobin concentrations, which in turn results in increased erythropoiesis under influence of increased production of erythropoietin. Red cell destruction may occur as a result of direct action by malaria parasites, or because a malaria-induced immune response leads to lysis of parasitized and nonparasitized red cells. ${ }^{22}$ It cannot be ruled out that, in addition, serum sTfR concentration is increased in malaria because of ineffective erythropoiesis, whereby developing erythroblasts die prematurely in the marrow without producing mature red cells. ${ }^{9}$

Our findings indicate that malaria-associated anemia, iron demand, and inflammation are greater in stunted than in nonstunted children. Although it is conceivable that malaria causes stunting, we consider it more likely that nutritional inadequacies that result in stunting also exacerbate the effects of malaria, given that malarial infection is a transient condition, and stunting becomes manifest only after a prolonged period of slowing in skeletal growth. Some of these nutritional inadequacies - such as deficiencies in zinc, ${ }^{23}$ iron $^{24,25}$ and possibly vitamin $\mathrm{A}^{26,27}$-are also known or suspected to impair host immunity. ${ }^{28-32}$ As reviewed by Scrimshaw et al, ${ }^{33}$ there is compelling evidence that reduced immunity due to nutritional deficiencies reduce host capacity to resist the consequences of a wide range of infections. This most likely explains the effects of malaria as observed in our study are more severe in stunted children than in their nonstunted counterparts.

Before 1950 it was widely accepted, although little supported by epidemiologic evidence, that malnutrition is associated with greater frequency and severity of malaria. ${ }^{34} \mathrm{~A}$ number of influential studies subsequently appeared that in fact suggested that malnourished children are to some degree protected against malaria. However, Shankar ${ }^{34}$ in a review concluded that these studies suffered from methodologic shortcomings and that the available evidence indicates that malnutrition is associated with increased occurrence of infection and symptomatic malaria, and considerably higher likelihood of malaria mortality in humans. This is in line with our findings, and implies that nutritional interventions using micronutrients may not only reduce stunting and nutritional anemia, but also reduce malaria-associated anemia. Famine or starvation may be a notable exception, because there is strong evidence that refeeding of starved individuals with latent infections carries an increased risk of symptomatic malaria. This may suggest that parasite proliferation following refeeding temporarily outpaces development of protective immunity. ${ }^{34}$ We could not demonstrate an influence of wasting on malaria or malaria-associated anemia (data not shown), perhaps because few of the children studied were wasted.

\section{CONCLUSION}

Our results give observational support to the proposed hypothesis that the nutritional inadequacies that cause stunting also impair host immunity, thus increasing the degree to which malaria is associated with decreased concentrations of hemoglobin, with increased inflammation and with increased iron required for developing erythroblasts. Increased intake of micronutrients may not only reduce stunting and nutritional anemia, but also reduce malaria-associated anemia. Increased intake of micronutrients may not only reduce stunting and nutritional anemia, but also reduce malaria-associated anemia. However, 
longitudinal studies are required to confirm causality.

\section{ACKNOWLEDGMENTS}

This study was funded through a grant from the Netherlands Foundation for the Advancement of Tropical Research (NWO/ WOTRO; grant WV93-273).

Hans Verhoef, Clive West, and Frans Kok were responsible for study design and interpretation of results. Hans Verhoef was responsible for data collection and analysis. Jacobien Veenemans and Yves Beguin assisted in interpretation of results, and Yves Beguin conducted the biochemical analyses. Yves Beguin is a Research Director of the National Fund for Scientific Research (FNRS, Belgium)

We thank Paul Ndeto, Stella Maweu, Obed Mwendwa, Joshua Kimanthi, Fred Kilonzo, Silas Nzyuko, local government authorities and auxiliary health workers, and the staff of the Ministry of Health at Kibwezi Rural Health Clinic for field and laboratory assistance; and the African Medical Research Foundation staff in Kibwezi for hosting this project.

\section{REFERENCES}

1. Ulijaszek SJ. Between-population variation in pre-adolescent growth. Eur J Clin Nutr. 1994;48(suppl 1):S5-S14

2. Waterlow JC. Causes and mechanisms of linear growth retardation (stunting). Eur J Clin Nutr. 1994;48(suppl 1):S1-S4

3. Tomkins A. The risk of morbidity in a stunted child. In: Waterlow JC ed. Linear Growth Retardation in Less Developed Countries. Nestlé Nutrition Workshop Series. Vevey, Switzerland: Nestec/New York, NY: Raven Press; 1988:185-199

4. Snow RW, Marsh K. New insights into the epidemiology of malaria relevant for disease control. Br Med Bull. 1998;54:293-309

5. Hendrickse RG, Hasan AH, Olumide LO, Akinkunmi A. Malaria in early childhood. An investigation of five hundred seriously ill children in whom a "clinical" diagnosis of malaria was made on admission to the children's emergency room at University College Hospital, Ibadan. Ann Trop Med Parasitol. 1971;65:1-20

6. McGregor IA. Malaria: nutritional implications. Rev Infect Dis. 1982;4: 798-804

7. Murray MJ, Murray NJ, Murray AB. Murray MB. Refeeding-malaria and hyperferraemia. Lancet. 1975;1:653-654

8. Feelders RA, Kuiper-Kramer EP, van Eijk HG. Structure, function and clinical significance of transferrin receptors. Clin Chem Lab Med. 1999; 37:1-10

9. Verhoef H, West CE, Ndeto P, Burema J, Beguin Y, Kok FJ. Serum transferrin receptor concentration indicates increased erythropoiesis in Kenyan children with asymptomatic malaria. Am J Clin Nutr. 2001;74: 767-775

10. How to Weigh and Measure Children: Assessing the Nutritional Status of Young Children in Household Surveys. New York, NY: United Nations, Department of Technical Cooperation for Development and Statistical Office, National Household Survey Capability Programme; 1986

11. Lohman TG, Roche AF, Martorell R. Anthropometric Standardization Reference Manual. Champaign, IL: Human Kinetics Books; 1988

12. Bennett S, Woods T, Liyanage WM, Smith DL. A simplified general method for cluster-sample surveys of health in developing countries. World Health Stat Q. 1991;44:98-106

13. Brogan D, Flagg EW, Deming M, Waldman R. Increasing the accuracy of the Expanded Programme on Immunization's cluster survey design. Ann Epidemiol. 1994;4:302-311

14. Dean AG, Dean JA, Coulombier D, et al. The Epi Info Manual, Version 6.02: A Word Processing, Database and Statistics System for Public Health on IBM-Compatible Microcomputers. London, England: Brixton Books; 1994

15. World Health Organization. Measuring Change in Nutritional Status: Guidelines for Assessing the Nutritional Impact of Supplementary Feeding Programmes for Vulnerable Groups. Geneva, Switzerland: World Health Organization; 1983

16. Nutrition and Health Status of Young Children and Their Mothers in Kenya: Findings From the 1993 Kenya Demographic and Health Survey. Calverton, MD: Macro International; 1996

17. Keller W. The epidemiology of stunting. In: Waterlow JC, ed. Linear Growth Retardation in Less Developed Countries. Nestlé Nutrition Workshop Series. Vevey: Nestec/New York: Raven Press; 1988:17-39

18. Victora CG. The association between wasting and stunting: an international perspective. J Nutr. 1992;122:1105-1110

19. DeMaeyer E, Adiels Tegman M. The prevalence of anaemia in the world. World Health Stat Q. 1985;38:302-316

20. WHO Working Group. Use and interpretation of anthropometric indicators of nutritional status. Bull World Health Organ. 1986;64:929-941

21. Weiss G. Iron and anemia of chronic disease. Kidney Int Suppl. 1999;69: S12-S17

22. Menendez C, Fleming AF, Alonso PL. Malaria-related anaemia. Parasitol Today. 2000;16:469-476

23. Umeta M, West CE, Haidar J, Deurenberg P, Hautvast JG. Zinc supplementation and stunted infants in Ethiopia: a randomised controlled trial. Lancet. 2000;355:2021-2026

24. Angeles IT, Schultink WJ, Matulessi P, Gross R, Sastroamidjojo S. Decreased rate of stunting among anemic Indonesian preschool children through iron supplementation. Am J Clin Nutr. 1993;58:339-342

25. Adish AA, Esrey SA, Gyorkos TW, Jean-Baptiste J, Rojhani A. Effect of consumption of food cooked in iron pots on iron status and growth of young children: a randomised trial. Lancet. 1999;353:712-716

26. Muhilal, Permeisih D, Idjradinata YR, Muherdiyantiningsih, Karyadi D. Vitamin A-fortified monosodium glutamate and health, growth, and survival of children: a controlled field trial. Am J Clin Nutr. 1988;48: 1271-1276

27. Hadi H, Stoltzfus RJ, Dibley MJ, et al. Vitamin A supplementation selectively improves the linear growth of Indonesian preschool children: results from a randomized controlled trial. Am J Clin Nutr. 2000;71:507-513

28. Brock JH. Iron in infection, immunity, inflammation and neoplasia. In: Brock JH, Halliday JW, Pippard MJ, Powell LW, eds. Iron Metabolism in Health and Disease. London, England: WB Saunders Co; 1994:353-389

29. Dallman PR. Iron deficiency and the immune response. Am J Clin Nutr. 1987:46:329-334

30. Salgueiro MJ, Zubillaga M, Lysionek A, et al. Zinc status and immune system relationship: a review. Biol Trace Elem Res. 2000;76:193-205

31. Semba RD. The role of vitamin A and related retinoids in immune function. Nutr Rev. 1998;56:S38-S48

32. Shankar AH, Prasad AS. Zinc and immune function: the biological basis of altered resistance to infection. Am J Clin Nutr. 1998;68(suppl): 447S-463S

33. Scrimshaw NS, Taylor CE, Gordon JE. Interactions of Nutrition and Infection. Geneva, Switzerland: World Health Organization; 1968

34. Shankar AH. Nutritional modulation of malaria morbidity and mortality. J Infect Dis. 2000;182(suppl 1):S37-S53 